\title{
Nonlinear localized modes in two-dimensional electrical lattices
}

\author{
L. Q. English, ${ }^{1}$ F. Palmero, ${ }^{2}$ J. F. Stormes, ${ }^{1}$ J. Cuevas, ${ }^{3}$ R. Carretero-González,${ }^{4}$ and P. G. Kevrekidis ${ }^{5}$ \\ ${ }^{1}$ Department of Physics and Astronomy, Dickinson College, Carlisle, Pennsylvania 17013, USA \\ ${ }^{2}$ Nonlinear Physics Group, Escuela Técnica Superior de Ingeniería Informática, Departamento de Física Aplicada I, \\ Universidad de Sevilla, Avenida Reina Mercedes s/n, 41012 Sevilla, Spain \\ ${ }^{3}$ Nonlinear Physics Group, Escuela Politécnica Superior, Departamento de Física Aplicada I, Universidad de Sevilla, \\ Calle Virgen de África 7, 41011 Sevilla, Spain \\ ${ }^{4}$ Nonlinear Dynamical Systems Group, Department of Mathematics and Statistics, and Computational Science Research Center, \\ San Diego State University, San Diego, California 92182-7720, USA \\ ${ }^{5}$ Department of Mathematics and Statistics, University of Massachusetts, Amherst, Massachusetts 01003-4515, USA
}

(Received 9 February 2013; revised manuscript received 15 May 2013; published 12 August 2013)

\begin{abstract}
We report the observation of spontaneous localization of energy in two spatial dimensions in the context of nonlinear electrical lattices. Both stationary and moving self-localized modes were generated experimentally and theoretically in a family of two-dimensional square as well as honeycomb lattices composed of $6 \times 6$ elements. Specifically, we find regions in driver voltage and frequency where stationary discrete breathers, also known as intrinsic localized modes (ILMs), exist and are stable due to the interplay of damping and spatially homogeneous driving. By introducing additional capacitors into the unit cell, these lattices can controllably induce mobile discrete breathers. When more than one such ILMs are experimentally generated in the lattice, the interplay of nonlinearity, discreteness, and wave interactions generates a complex dynamics wherein the ILMs attempt to maintain a minimum distance between one another. Numerical simulations show good agreement with experimental results and confirm that these phenomena qualitatively carry over to larger lattice sizes.
\end{abstract}

DOI: 10.1103/PhysRevE.88.022912

PACS number(s): 05.45.Yv, 63.20.Pw, 63.20.Ry

\section{INTRODUCTION}

It has long been known that solitons emerge as classes of solutions to many nonlinear (lattice and partial) differential equations described chiefly by one spatial dimension; prominent examples are the KdV equation, the sine-Gordon equation, and the nonlinear Schrödinger equation. In two dimensions, quasi one-dimensional localization patterns can often still occur [1], but robust two-dimensional (2D) localization in continuous media is rather atypical (see Ref. [2] and references therein). However, it is well-known that the discreteness of the underlying medium allows for the existence of discrete breathers, also known as intrinsic localized modes (ILMs), in one-dimensional [3] and even in higher dimensional lattices [4,5]. Alternatively, one can externally enforce or introduce a periodicity in the form of a regular modulation in some property of the continuous (e.g., in optical photorefractive crystal or atomic Bose-Einstein condensate) media [6,7], thus again breaking continuous translational symmetry. Such nonlinear optics and atomic physics [8] settings are the prototypical ones where stable localized solitary wave structures have been reported.

Here we show that two-dimensional discrete breathers experimentally exist and are stable in the context of twodimensional, damped-driven electrical lattices. We characterize these breather and multibreather states in parameter space, and we compare them to the results of numerical simulations and stability analysis. Finally, we focus particularly on versions of these lattices that support moving breathers. Discrete breathers have been considered in a variety of other settings experimentally including (but not limited to) micromechanical cantilever arrays [9], chains of coupled pendula [10], Josephson-junction ladders (JJLs) [11], granular crystals of beads interacting through Hertzian contacts [12], layered antiferromagnetic crystals [13], halide-bridged transitionmetal complexes [14], and dynamical models of the DNA double strand [15]. Yet, in most of these examples, the coherent structures are effectively one-dimensional. Even when higher dimensional (as is, e.g., possible in optical waveguide arrays or photorefractive crystals [6]), the states are typically stationary. Hence, the experimental ability to systematically generate discrete breathers in two-dimensional electrical lattices and, perhaps especially, to control their mobility launches a new dimension in the modeling, and the theoretical and experimental understanding of such states. This may be of broader interest in other areas as well, such as JJLs (see, e.g., the theoretical proposal of Ref. [16]) and two-dimensional granular crystals (see, e.g., the recent experimental realization of Ref. [17]). Another attractive feature of our lattices is their potential square or honeycomb geometry, especially since the latter has been a point of intense investigation at both the linear and the nonlinear level. This is due to its conical diffraction and Dirac (diabolical) points examined intensely in both the physical $[18,19]$ and mathematical [20] communities, as well as due to potential connections with graphene nanoribbons; see, e.g., Refs. [21,22] and references therein.

\section{EXPERIMENTAL AND THEORETICAL SETUP}

The experimental system under investigation is a class of two-dimensional electrical lattices of either honeycomb or square geometry, as shown in Fig 1. These electrical lattices can be considered as a set of single cells representing nonlinear LC oscillators, each composed of a varactor diode (NTE 618 ) with a nonlinear capacitance $C(V)$ and an inductor $L_{2}=330 \mu \mathrm{H}$. These single cells are then coupled at point $B$ by inductors $L_{1}=680 \mu \mathrm{H}$ and are driven by a single sinusoidal voltage source $V(t)$, which is identical for all cells, 

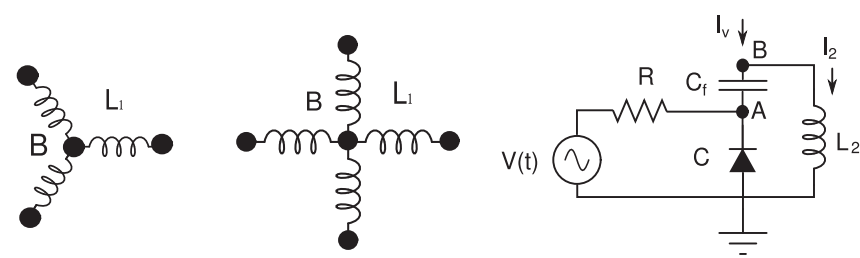

FIG. 1. Left: Schematic circuit diagrams of the basic geometry of the two electrical transmission lines (honeycomb and square), where black points represent single elements (right), with or without the block capacitor $C_{f}$. Every single element is connected to a sinusoidal voltage source $V(t)$ via a resistor $R$, and grounded. A good way of visualizing the full lattice is to start with a plane of inductors by extending either of the two diagrams on the left into a honeycomb or square, respectively, periodic pattern. Then, each vertex B of this inductor plane attaches (via wires) to point B copies of the right diagram (one for each vertex), and these copies are oriented perpendicular to the inductor plane.

via a resistor $R=10 \mathrm{k} \Omega$, with amplitude $V_{d}$ and frequency $f$. We study two different unit cell versions, with the only difference being the presence of a block capacitor between the diode and the coupling inductor in one of them, as explained in Ref. [23]. The effect of the block capacitor is to make ILMs mobile; here we study two different values of block capacitances, $C_{f}=1 \mu \mathrm{F}$ and $C_{f}=15 \mathrm{nF}$. The experimental lattices were composed of 36 elements with periodic boundary conditions, and node voltages $V_{n, m}$ at points $B$ were measured at a rate of $2.5 \mathrm{MHz}$ using a multichannel analog-to-digital converter. In our experimental setup, we enforce periodic boundary conditions by connecting each boundary element with its corresponding one on the other side of the lattice via inductors $L_{1}$.

In the linear limit of small-amplitude plane waves, we have obtained the dispersion relation, which yields as the lowest frequency (uniform mode) $f_{\text {min }} \cong 312 \mathrm{kHz}$, and the highest frequency $f_{\max } \cong 689 \mathrm{kHz}$ in the square geometry configuration, and $f_{\text {max }} \cong 617 \mathrm{kHz}$ in the honeycomb lattice. Notice that this highest-frequency mode in the linear spectrum is now above the second harmonic of the uniform mode in square geometry lattice, and below it, but very close, in the honeycomb configuration.

Using basic circuit theory, in the block capacitor case, and a procedure similar to the one used in the corresponding onedimensional electrical line (see Ref. [24] for more details), the dynamics of the lattice can be described by the equations

$$
\begin{aligned}
\frac{d i_{n, m}}{d \tau}= & \frac{L_{2}}{L_{1}}\left(\sum_{j, k} v_{j, k}^{B}-q v_{n, m}^{B}\right)-v_{n, m}^{B}, \\
\frac{d v_{n, m}^{A}}{d \tau}= & \frac{1}{c\left(v_{n, m}^{A}\right)}\left[i_{n, m}-i^{D}\left(v_{n, m}^{A}\right)-\frac{v_{n, m}^{B}}{C_{0} \omega_{0} R_{l}}\right. \\
& \left.+\frac{\cos (\Omega \tau)}{C_{0} \omega_{0} R}-\frac{v_{n, m}^{A}}{C_{0} \omega_{0} R}\right], \\
\frac{d v_{n, m}^{B}}{d \tau}= & \frac{d v_{n, m}^{A}}{d \tau}+\frac{C_{0}}{C_{f}}\left[i_{n, m}-\frac{v_{n, m}^{B}}{C_{0} \omega_{0} R_{l}}\right],
\end{aligned}
$$

where the sum is taken over first, $q$, neighbors, $q=4$ in square lattice case and $q=3$ in honeycomb one. Also, the following dimensionless variables were used: $\tau=\omega_{0} t$; $i_{n, m}=\left(I_{v}-I_{2}\right) /\left(\omega_{0} C_{0} V_{d}\right)$, where $I_{v}$ is the full current through the unit cell and $I_{2}$ the current through the inductor $L_{2}$, both corresponding to cell $(n, m) ; v=V / V_{d}$, used with superscripts $\mathrm{A}$ and $\mathrm{B} ; V_{n, m}^{B}$ is the measured voltage at node $(n, m) ; V_{n, m}^{A}$ is the voltage at an intermediate point between the varactor and the block capacitor; $\Omega=2 \pi f / \omega_{0}, \omega_{0}=1 / \sqrt{L_{2} C_{0}} ; i_{D}=$ $I_{D} /\left(\omega_{0} C_{0} V_{d}\right)$, where $I_{D}$ is the current through the varactor diode; $c=C(V) / C_{0}$, where $C_{0}=C(0)$ and $C(V)$ is the nonlinear capacitance of the diode. Also, a phenomenological dissipation resistor, $R_{l}$, was included in the model to better approximate the experimental dynamics, and its value has been determined by matching the numerical and experimental nonlinear resonance curves corresponding to a single element [24]. A simplified set of equations corresponding to the nonblock capacitor case can be obtained by considering the limit $C_{f} \rightarrow \infty$. In all cases, the ratio $L_{2} / L_{1}$ characterizes the strength of the "effective" discreteness of the system.

\section{STATIONARY REGULAR AND SUBHARMONIC DISCRETE BREATHERS IN SQUARE AND HONEYCOMB 2D LATTICES}

In order to find ILMs in Eq. (1), we have employed the well-known modulational instability of the driven uniform mode $[4,25]$. The stability analysis is performed by getting an exact periodic orbit by means of a shooting method [26], and subsequently performing a Floquet analysis [27]. In the case without block capacitors, we obtain stationary and stable one-peak breathers, as shown in Fig. 2. These localized modes are robust, persisting as long as the driver remains on.

Our numerics are generally found to be in good agreement with experimental results. Yet, it can be seen in Fig. 2 that the amplitude of the one-peak breather is slightly higher in the numerics than in the experimental data. We attribute this slight mismatch to energy dissipation in the coupling inductors; these are stronger in the square lattice setting than in the honeycomb geometry, and they are not taken into account in our simplified model of Eq. (1). When this dissipation is included in our model, by means of some phenomenological small resistances in series with inductors $L_{1}$, this further improves the agreement for the ILM amplitude. For even higher amplitudes in the experiment, multipeak breathers have been observed, which, in most cases, can also be captured essentially by the model. In these cases, experimental data suggest that impurities play a very important role for the stabilization and the localization of peaks, as can be appreciated also in the comparison with numerical simulations (see Fig. 3).

In particular, although the experiments display multipeak (two-peak) breathers in $6 \times 6$ square lattices, our numerics tend to suggest that such multipeak breathers do not exist as exact periodic orbits in the numerical model for square lattices smaller than $8 \times 8$. In fact, even in the $8 \times 8$ case the two-peak breathers found therein are slightly unstable as is evidenced from the corresponding numerics shown in Fig. 3. For larger lattices these two-peak breathers become stable and for even larger lattices higher multipeak breathers can be supported (results now shown here).

As in the one-dimensional line [28], we again observe subharmonic breathers (experimentally and theoretically) in 

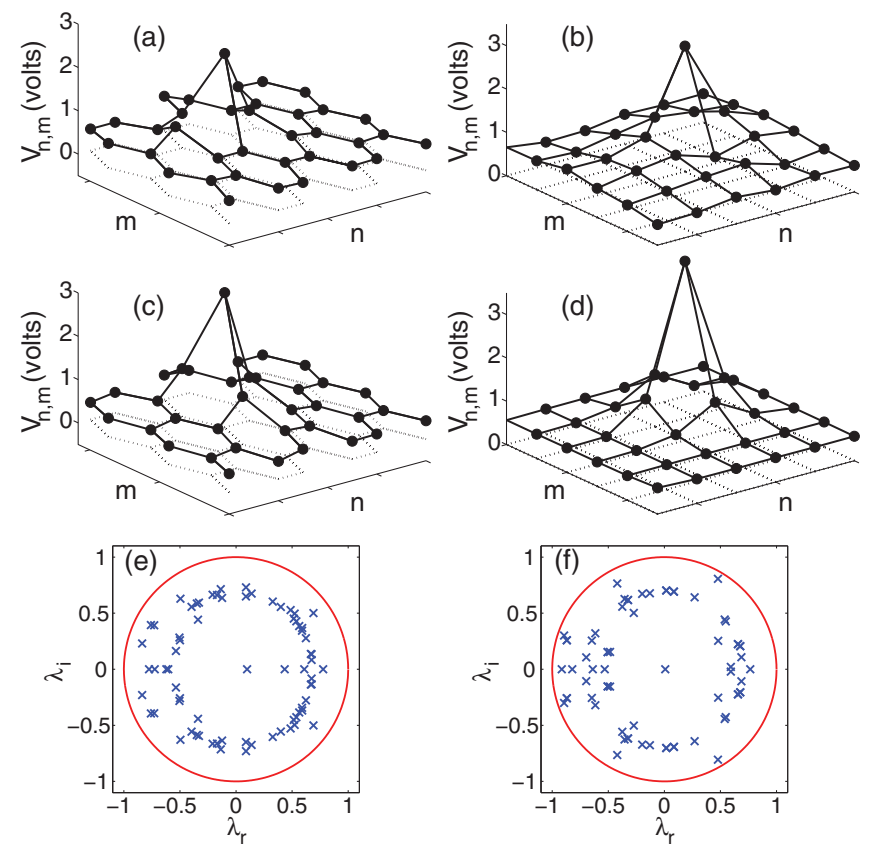

FIG. 2. (Color online) Comparison between the experimental [(a) and (b)] and numerical [(c) and (d)] profiles of stationary one-peak breathers in a honeycomb lattice, (a) and (c), and a square lattice, (b) and (d), in the $(n, m)$ plane. The bottom panels show the Floquet numerical linearization spectrum corresponding to the honeycomb lattice (e), and the square lattice (f), confirming (since all multipliers are inside the unit circle) the stability of solutions. The driver has amplitude $V_{d}=1.5 \mathrm{~V}$ and frequency $f=283 \mathrm{kHz}$ in the honeycomb lattice case, and $V_{d}=2 \mathrm{~V}$ and $f=290 \mathrm{kHz}$ (experimental) and $f=301 \mathrm{kHz}$ (numerical) in the square lattice case (in the latter setting, our computations show the one-peak breather at $290 \mathrm{kHz}$ to be weakly unstable and for this reason we have selected to show this very proximal frequency value for which the breather is dynamically robust).

two dimensions. In Fig. 4, the peak of the breather oscillates with a frequency $f_{\text {ILM }}=f / 2$. Notably, in two dimensions it is generally more difficult to stabilize breathers via subharmonic driving - the driving conditions have to fall into a fairly limited region of parameter space.

\section{MOVING DISCRETE BREATHERS IN SQUARE AND HONEYCOMB 2D LATTICES}

When a block capacitor of $1 \mu \mathrm{F}$ is placed in series with the diode in the honeycomb or square lattice, the static breathers become mobile, as was the case for the one-dimensional chain [23]. However, in the subharmonic breather case, the introduction of that same type of block capacitor has a detrimental effect on the breather-a feature which is qualitatively distinct from the corresponding one-dimensional lattice for which mobile subharmonic breathers were reported. The fact that the frequency of the subharmonic driver, namely twice the ILM response frequency, is now positioned within, or very close to, the linear dispersion band of the $2 \mathrm{D}$ lattice may explain this fragility of the $2 \mathrm{D}$ subharmonic breather.
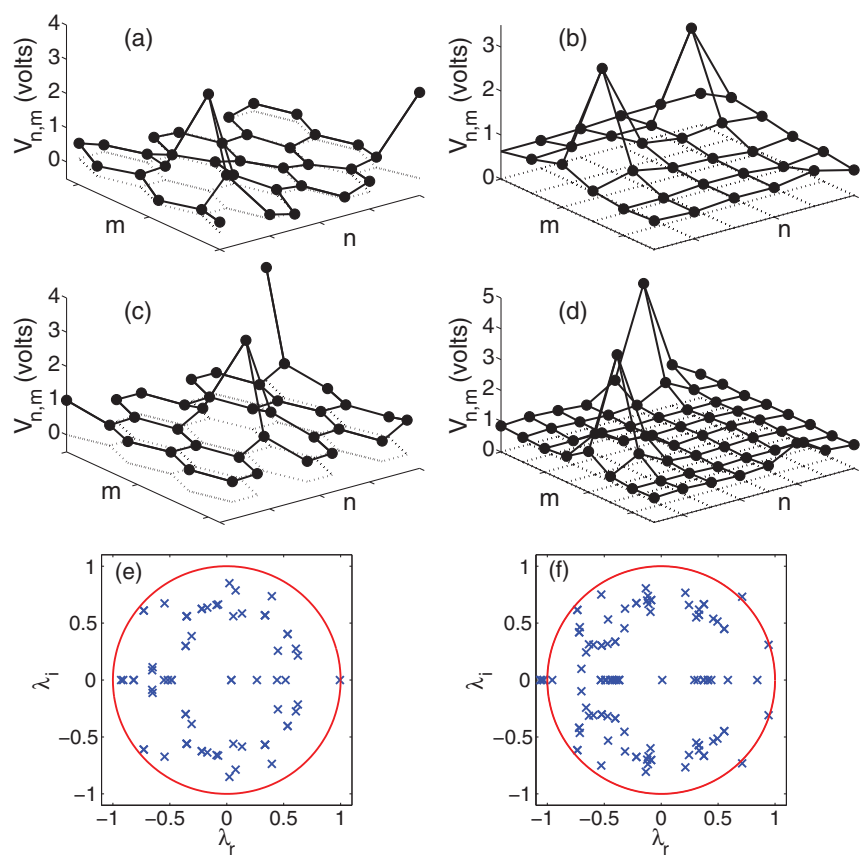

FIG. 3. (Color online) Comparison between the experimental [(a) and (b)] and numerical [(c) and (d)] profiles of stationary multipeak breathers in a honeycomb lattice, (a) and (c), and a square lattice, (b) and (d), in the $(n, m)$ plane. The bottom panels show the Floquet numerical linearization spectrum corresponding to the honeycomb lattice (e), and the square lattice (f). The driver has amplitude $V_{d}=$ $2.5 \mathrm{~V}$ and frequency $f=298 \mathrm{kHz}$ (experimental) and $f=301 \mathrm{kHz}$ (numerical) in the honeycomb lattice case and $V_{d}=3.0 \mathrm{~V}$ and $f=300 \mathrm{kHz}$ (experimental and numerical) in the square lattice case. For the case of square lattices smaller than $8 \times 8$, multipeak breathers were not found in our numerics. Therefore, we present the results for an $8 \times 8$ lattice where two-peak breathers were indeed found as exact solutions (although slightly unstable as the Floquet multipliers show).

Nevertheless, and contrary to the simpler situation in one dimension, where a clear direction of movement arises, the motion through the lattice appears to be more complex, as shown in Fig. 5. In general, numerical simulations obtained solving Eq. (1) show fairly good agreement with experiments and yield information about the intrinsic nature of this complex
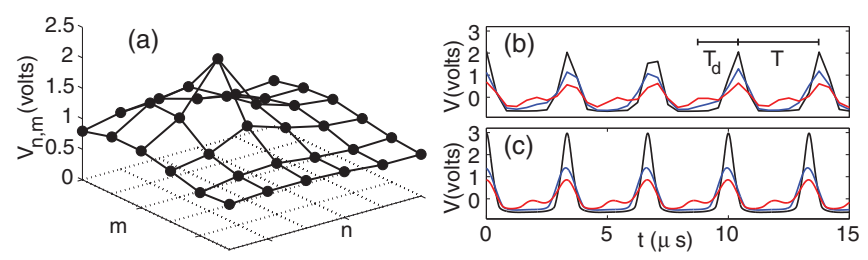

FIG. 4. (Color online) Subharmonic breather corresponding to a square lattice, $V_{d}=6.4 \mathrm{~V}$ and $f=600 \mathrm{kHz}$ in the $(n, m)$ plane. (a) Experimental spatial profile, and (b) experimental and (c) numerical temporal oscillations of three different nodes: the black (upper) line corresponds to the ILM center, the blue (middle) line corresponds to the first neighbor, and the red (lower) line corresponds to the node farthest away from the ILM center. Note that the oscillations of the node farthest away from the ILM center display local maxima corresponding to the natural period $T$ of the ILM at twice the driving period $T_{d}=1 / f$. 

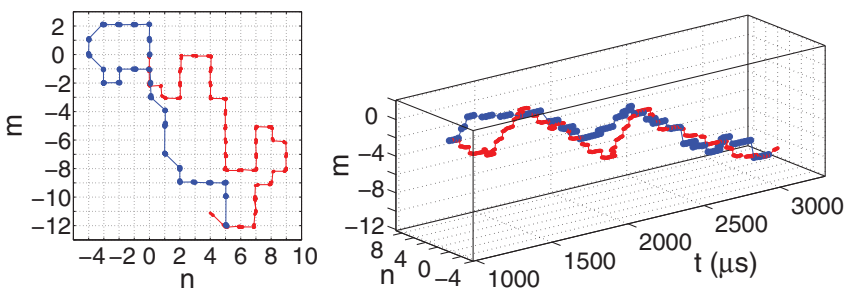

FIG. 5. (Color online) Trajectory of a one-peak moving breather on a square lattice for $V_{d}=2 \mathrm{~V}, f=300 \mathrm{kHz}$, and $C_{f}=1 \mu \mathrm{F}$. Depicted are the positions of the center of mass of the breather in the $(n, m)$ plane (left) and $(n, m, t)$ space (right). The small (red) dots depict the experimental results and the large (blue) dots depict the corresponding numerical simulations. The trajectories have been unwrapped from the periodic lattice. Solid lines have been added for guidance.

motion, where a strong sensitivity to small inhomogeneities (i.e., lattice defects) is seen. The transition from one node to the next is such that after a number of periods a noticeable asymmetry develops in the ILM profile; this leads to two neighboring nodes attaining equal amplitude, and finally the ILM becoming centered (initially between two sites and then) on the next node. This sequence is now also observed in two dimensions. Notice that the existence and mobility problem of ILMs has been argued to be quite important in other nonsquare lattices, such as the hexagonal one where breathers were proposed as responsible for the presence of dark lines in natural crystals of muscovite mica [29], and reconstructive transformations in layered silicates [30].

Contrary to the one-dimensional lattices, where mobile breathers can travel along very large lattices [31], in our square electrical lattice, we generally observe (cf. Fig. 5) a directed motion which is interrupted by (longer) intervals of localization as the wave struggles to overcome the well-known Peierls-Nabarro (PN) barrier [4]. Note that given the small size of the lattice, in addition to the role of inhomogeneities, small amplitude residual excitations ("phonons") are also important in directing the breather motion. These features, while present in the experiment, cannot be quantified in a way that would enable a quantitative comparison with the numerical computation of the associated dynamics. It is thus expected that the agreement in this part of the work will be qualitative but not quantitative between theory/computation and experimental findings. It is also worth mentioning that, although the movement along the square lattice usually happens along the edges of the lattice, some transitions are also observed to happen, both in the experiment and the numerics, along the diagonals that have a slightly higher PN barrier. Despite the complex nature of the motion of the breathers, the organizing principle obeyed in both one and two dimensions is that the ILM never hops back to the node it occupied prior to its current location due to some residual capacitor-charge impurity associated with that node. In one dimension, this principle necessarily gives rise to orderly, unidirectional motion. In two-dimensional hexagonal lattices there are still two choices available to the ILM, and in a square lattice there are three. Thus, the motion does not have to be unidirectional. It would be interesting to statistically measure the "biased diffusion" associated with the apparent irregular trajectories displayed by the moving

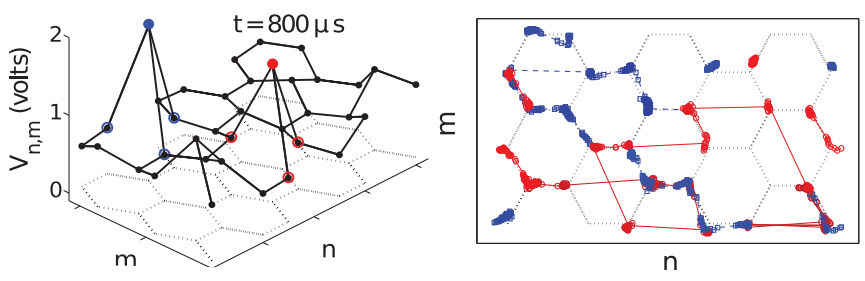

FIG. 6. (Color online) Left: Experimental two-peak moving breather profile on a honeycomb lattice with periodic boundary conditions, in the $(n, m)$ plane. The blue circles (dark gray) correspond to the center of mass of one peak and the red circles (light gray) to the center of mass of the other one, for $V_{d}=2.5 \mathrm{~V}, f=330 \mathrm{kHz}$, and $C_{f}=1 \mu \mathrm{F}$. Right: Trajectory of the corresponding centers of mass of the two interacting breathers (circles [red] and squares [blue]). Solid lines have been added for guidance.

breathers. Although the system is deterministic, due to small defects and residual excitations, the breathers seem to follow a biased erratic motion with the constraint of not going back in the direction they came from. Comparing the scaling of the diffusion associated with this peculiar behavior with the classical Brownian motion and biased random walk variants thereof would be an interesting avenue for future exploration. Another interesting direction of future work is to find out how this biased erratic motion results from the interplay of the nonlinearity with the damped and driven nature of our lattice and, critically (since 1d analogs of our lattice did not feature such motion typically) with the higher dimensionality. A comparison of such findings with the ones from one-dimensional lattices featuring damped-driven, potentially chaotic breather dynamics [25,32] would also be quite meaningful.

In general, multipeak breathers, or collections of breathers, also become mobile in the lattices with block capacitors. In all of our experimental results, the relevant motion is complex yet the relative distance between peaks remains fairly constant and does not fall below a minimum value (two edges away) (cf. Fig. 6). This observation suggests that these two ILMs tend to repel each other upon close proximity in this small lattice. We also note, as in the square lattice, that although most transitions during motion happen through the lattice edges, there are some transitions that happen along the long diagonal of the honeycomb cell (cf. Fig. 6). We note that we did not observe transitions along the short diagonals of the honeycomb cell.

A different scenario takes place when a smaller block capacitance, $C_{f}=15 \mathrm{nF}$, is chosen. In that case, breathers become mobile but quickly collapse into a collective pattern resembling a planar (one-dimensional) wavefront, which rapidly transports energy coherently through the system, as shown in Fig. 7. It is intriguing that a spatially homogeneous driver
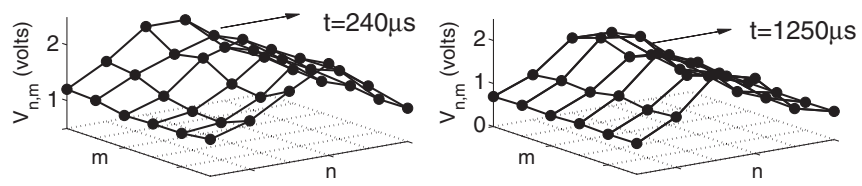

FIG. 7. Experimental (left) and numerical (right) moving front profile, in the $(n, m)$ plane, for a $6 \times 6$ square lattice. $V_{d}=2 \mathrm{~V}$, $f=300 \mathrm{kHz}$, and $C_{f}=15 \mathrm{nF}$. 
can sustain such nonlinear collective patterns characterized by energy transport. We should point out that the types of coherent patterns experimentally observable in the honeycomb lattice are apparently more complex than in the square lattice where only true planar wavefronts are seen. For the honeycomb lattice the equivalent planar waves can be found at particular driving frequencies and amplitudes (results not shown here). Numerical simulations performed on larger square lattices (results not shown here) suggest that onedimensional wavefront profiles are not robust-presumably due to transverse instabilities - and break down into patterns consisting of several ILMs, each one extending over a number of sites.

\section{CONCLUSIONS AND FUTURE CHALLENGES}

In summary, we have generated two-dimensional discrete breathers in the setting of damped-driven electrical lattices. To our knowledge, this is the first time that breathers have been experimentally stabilized in higher-dimensional discrete lattices by direct (and subharmonic) driving, with motion systematically induced to them and that these features have been examined not only in square but importantly also in honeycomb lattices.

We have characterized the statics, stability, and mobility of these modes of self-localized energy, centered over a particular node of the lattice, and extending over a few lattice sites. The breathers have been found to persist indefinitely, and are either stationary or hopping in the lattice, depending on the precise makeup of the unit cell. We have observed that interacting breathers seem to repel each other when placed in close proximity which has the effect of (roughly) maintaining their pairwise distance in our case example of small honeycomb periodic electrical lattices.

This work paves the way for numerous studies. These include the detailed characterization of breathers, their stability and mobility properties and associated potential barrier in long (and infinite) 2D chains, as well as their pairwise interactions; the characterization of impurities and their role in inducing mobility; the examination of spectral gap and higher gap (nonlinear) states [33], especially in honeycomb lattices; and the generalization of such chains even in fully 3D configurations. These issues of broader interest to other settings such as Josephson-junction ladders [16] and granular crystals [17] will be considered in future publications.

\section{ACKNOWLEDGMENTS}

This research was supported by the Ministerio de Ciencia e Innovación of Spain (FIS2008-04848), University of Seville (plan propio), the US-NSF via CMMI-1000337, and USAFOSR via FA9550-12-1-0332. F.P. acknowledges Dickinson College for hospitality.
[1] M. J. Ablowitz and D. E. Baldwin, Phys Rev. E 86, 036305 (2012).

[2] K. Staliunas, R. Herrero, and G. J. de Valcárcel, Phys. Rev. A 75, 011604 (2007).

[3] A. J. Sievers and S. Takeno, Phys. Rev. Lett. 61, 970 (1988); J. B. Page, Phys. Rev. B 41, 7835 (1990); R. S. MacKay and S. Aubry, Nonlinearity 7, 1623 (1994).

[4] S. Flach and A. V. Gorbach, Phys. Rep. 467, 1 (2008).

[5] P. G. Kevrekidis, The Discrete Nonlinear Schrödinger Equation (Springer-Verlag, Heidelberg, 2009).

[6] F. Lederer, G. I. Stegeman, D. N. Christodoulides, G. Assanto, M. Segev, and Y. Silberberg, Phys. Rep. 463, 1 (2008).

[7] O. Morsch and M. Oberthaler, Rev. Mod. Phys. 78, 179 (2006).

[8] J. W. Fleischer, M. Segev, N. K. Efremidis, and D. N. Christodoulides, Nature (London) 422, 147 (2003);

B. Eiermann, Th. Anker, M. Albiez, M. Taglieber, P. Treutlein, K.-P. Marzlin, and M. K. Oberthaler, Phys. Rev. Lett. 92, 230401 (2004).

[9] M. Sato, B. E. Hubbard, and A. J. Sievers, Rev. Mod. Phys. 78, 137 (2006).

[10] J. Cuevas, L. Q. English, P. G. Kevrekidis, and M. Anderson, Phys. Rev. Lett. 102, 224101 (2009).

[11] P. Binder, D. Abraimov, A. V. Ustinov, S. Flach, and Y. Zolotaryuk, Phys. Rev. Lett. 84, 745 (2000); E. Trías, J. J. Mazo, and T. P. Orlando, ibid. 84, 741 (2000); J. J. Mazo and T. P. Orlando, Chaos 13, 733 (2003); A. V. Ustinov, ibid. 13, 716 (2003).
[12] N. Boechler, G. Theocharis, S. Job, P. G. Kevrekidis, M. A. Porter, and C. Daraio, Phys. Rev. Lett. 104, 244302 (2010).

[13] L. Q. English, M. Sato, and A. J. Sievers, Phys. Rev. B 67, 024403 (2003); U. T. Schwarz, L. Q. English, and A. J. Sievers, Phys. Rev. Lett. 83, 223 (1999).

[14] B. I. Swanson, J. A. Brozik, S. P. Love, G. F. Strouse, A. P. Shreve, A. R. Bishop, W.-Z. Wang, and M. I. Salkola, Phys. Rev. Lett. 82, 3288 (1999).

[15] M. Peyrard, Nonlinearity 17, R1 (2004).

[16] J. J. Mazo, Phys. Rev. Lett. 89, 234101 (2002).

[17] A. Leonard and C. Daraio, Phys. Rev. Lett. 108, 214301 (2012).

[18] M. J. Ablowitz, S. D. Nixon, and Y. Zhu, Phys. Rev. A 79, 053830 (2009).

[19] O. Bahat-Treidel, O. Peleg, M. Segev, and H. Buljan, Phys. Rev. A 82, 013830 (2010).

[20] C. L. Fefferman and M. I. Weinstein, J. Am. Math. Soc. 25, 1169 (2012).

[21] A. V. Savin and Yu. S. Kivshar, Phys. Rev. B 81, 165418 (2010).

[22] M. I. Molina and Yu. S. Kivshar, Opt. Lett. 35, 2895 (2010).

[23] L. Q. English, F. P. Palmero, A. J. Sievers, P. G. Kevrekidis, and D. H. Barnak, Phys. Rev. E 81, 046605 (2010).

[24] F. Palmero, L. Q. English, J. Cuevas, R. Carretero-González, and P. G. Kevrekidis, Phys. Rev. E 84, 026605 (2011).

[25] J. L. Marín, F. Falo, P. J. Martínez, and L. M. Floría, Phys. Rev. E 63, 066603 (2001).

[26] J. L. Marín and S. Aubry, Nonlinearity 9, 1501 (1996); T. Cretegny and S. Aubry, Phys. Rev. B 55, R11929 (1997).

[27] S. Aubry, Physica D 261, 1 (1996). 
[28] L. Q. English, F. Palmero, P. Candiani, J. Cuevas, R. CarreteroGonzalez, P. G. Kevrekidis, and A. J. Sievers, Phys. Rev. Lett. 108, 084101 (2012).

[29] J. L Marín, J. C Eilbeck, and F. M Russell, Phys. Lett. A 248 , 225 (1998).

[30] J. F. R. Archilla, J. Cuevas, M. D. Alba, M. Naranjo, and J. M. Trillo, J. Phys. Chem. 110, 24112 (2006).
[31] S. Aubry and T. Cretegny, Physica D 119, 34 (1998); D. Chen, S. Aubry, and G. P. Tsironis, Phys. Rev. Lett. 77, 4776 (1996); R. S. MacKay and J. A. Sepulchre, J. Phys. A: Math. Gen. 35, 3985 (2002).

[32] P. J. Martínez, M. Meister, L. M. Floría, and F. Falo, Chaos 13, 610 (2003).

[33] B. Z. Essimbi and I. V. Barashenkov, J. Phys. Soc. Jpn. 71, 2061 (2002). 